\title{
CB1 receptor antagonist rimonabant protects against chronic intermittent hypoxia-induced bone metabolism disorder and destruction in rats
}

\author{
Zhan-jun Dou ${ }^{1}$ - Xiao-Ling Gao ${ }^{2}$ Y Yan-li Jia ${ }^{3} \cdot$ Jie Chen $^{2}$ • Jiao-Jiao Yang ${ }^{1} \cdot$ Yan Chen ${ }^{2} \cdot$ Shu-jie Wu ${ }^{4} \cdot$ Tao Liu $^{1}$. \\ Mei-ting Wang ${ }^{1} \cdot$ Chong Yang $^{1} \cdot$ Na Zhang $^{1} \cdot$ Bei Wang ${ }^{2}$ (i)
}

Received: 23 June 2019 /Revised: 19 December 2019 / Accepted: 23 December 2019 / Published online: 2 January 2020

(C) The Author(s) 2020

\begin{abstract}
Objective The endocannabinoid system (ECS) regulates bone turn-over and remodeling. Chronic intermittent hypoxia $(\mathrm{CIH})$ occurring during obstructive sleep apnea (OSA) may lead to disorders of the ECS and bone metabolism abnormalities. This study aimed to investigate whether or not the cannabinoid receptor 1 (CB1R) antagonist rimonabant (Ri) alleviates bone metabolism abnormalities and bone destruction induced by chronic intermittent hypoxia (CIH).

Methods Healthy male Sprague Dawley (SD) rats $(\mathrm{n}=48)$ were randomly divided into 6 groups of 8 rats: 2 normal control (NC) groups, 2 intermittent hypoxia (IH) groups, and $2 \mathrm{IH}+\mathrm{Ri}$ groups. Rats in $\mathrm{NC}$ groups breathed room air for 4 weeks $(4 \mathrm{w} \mathrm{NC}$ group) and 6 weeks (6w NC group). Rats in IH groups experienced IH environment for 4 weeks (4w IH group) and 6 weeks ( $6 \mathrm{w}$ IH group). In addition to the same IH exposure, rats in IH + Ri group were given daily intraperitoneal injection of Ri at the dosage of $1.5 \mathrm{mg} / \mathrm{kg} / \mathrm{d}$ for 4 weeks (4w IH + Ri group) and 6 weeks (6w IH + Ri group). Levels of serum tartrate-resistant acid phosphatase (TRAP, a marker of bone resorption) were determined by ELISA. Hematoxylin and eosin (HE) staining was performed on bone sections to observe the changes in bone microstructure. Expression of CB1R in bone tissue was determined by immunohistochemistry.

Results TRAP levels were higher in the 4w IH and 6w IH groups than in the 4w NC and 6w NC groups; TRAP levels were lower in the $4 \mathrm{w} \mathrm{IH}+\mathrm{Ri}$ and $6 \mathrm{w}$ IH + Ri groups than in the 4w IH and 6w IH groups. HE staining showed that the morphology of bone cells in the NC group was normal, but the $4 \mathrm{w}$ IH group had mild edema of bone cells, reduction in trabecular bone, and destruction of bone microstructure. Changes were more severe in the $6 \mathrm{w}$ IH group than $4 \mathrm{w}$ IH. The $4 \mathrm{w}$ IH + Ri group was slightly improved compared with the 4w IH group. The $6 \mathrm{w} \mathrm{IH} \mathrm{+} \mathrm{Ri} \mathrm{group} \mathrm{was} \mathrm{improved} \mathrm{compared} \mathrm{with} \mathrm{the} \mathrm{4w} \mathrm{IH} \mathrm{+} \mathrm{Ri} \mathrm{group.}$ The results of immunohistochemistry showed that the expression of CB1R in IH group was significantly higher than that in NC group. The expression of CB1R in the IH + Ri group was lower than that in the IH group. With the prolongation of hypoxia, the expression of CB1R in bone cells of IH group increased. The expression level of CB1R in IH + Ri group decreased with the prolongation of intervention time. Correlation analysis showed that the expression rate of CB1R in bone cells was positively correlated with the level of TRAP in serum.

Conclusion $\mathrm{CIH}$ increases serum TRAP levels and triggers metabolic bone disorder by activating bone CB1R. Intervention with $\mathrm{CB} 1 \mathrm{R}$ antagonist (rimonabant) reduces the bone dysmetabolism in the $\mathrm{CIH}$ rat model.
\end{abstract}

Keywords Obstructive sleep apnea syndrome (OSAS) - Cannabinoid receptor 1 (CB1R) · Tartrate-resistant acid phosphatase (TRAP) · Rimonabant (Ri) · Chronic intermittent hypoxia (CIH)

Bei Wang

myemaila@qq.com

1 Department of the Second Clinical Medicine, Shanxi Medical University, Taiyuan, Shanxi, People's Republic of China

2 Department of Respiratory and Critical Care Medicine, The Second Hospital of Shanxi Medical University, No. 382, Wuyi Road, Taiyuan 030001, Shanxi, People's Republic of China
3 Department of Respiratory, Linfen Centrol Hospital, Linfen, Shanxi, People's Republic of China

4 Medical Records Statistics Office, The First Hospital of Shanxi Medical University, Taiyuan, Shanxi, People's Republic of China 


\section{Introduction}

Obstructive sleep apnea syndrome (OSAS) causes chronic intermittent hypoxia $(\mathrm{CIH})$ along with a series of pathological conditions such as sympathetic system activation, oxidative stress, and a cascade of systemic inflammation [1]. The hypoxia-reoxygenation pattern seen with $\mathrm{CIH}$ is a main pathophysiological feature of OSAS [2] and may result in damage to a number of target organs [3]. Over time, OSAS is associated with numerous comorbid conditions including hypertension, hyperlipidemia, insulin resistance, abnormal glucose tolerance, obesity, asthma, myocardial infarction, heart failure, and stroke. Data from numerous countries have shown a very close correlation of OSAS with increasing age and double the prevalence of the disease in men compared to women [4-6]. A number of studies, including a systematic review of 24 studies published in 2017, estimate the overall prevalence of obstructive sleep apnea to be between $9 \%$ and $38 \%$ in the general adult population [7-9].

Accumulating evidence has shown that a hypoxic environment may cause abnormal changes in bone metabolism resulting in osteoporosis [10], a systemic metabolic bone disease characterized by inferior quality of bone and damage to bone microstructure with increased risk for bone fragility and fractures. Patients with OSAS may represent a group at risk for bone resorption and loss of bone mineral density. It is important to evaluate patients with OSAS for possible bone loss [11].

The endocannabinoid system (ECS) is an endogenous signaling system involved in the regulation of various physiological functions in vivo [12]. The ECS is composed of the cannabinoid receptors $\mathrm{CB} 1 \mathrm{R}$ and $\mathrm{CB} 2 \mathrm{R}$ and their degradation enzyme and synthetase. These two receptors are $G$ protein-coupled receptors [13]. CB1R plays an important role in adjusting bone mass and bone remodeling. [14-16]. A previous clinical study by this research group showed that patients with OSAS may develop a disorder of the ECS, which may be relieved by continuous positive airway pressure (CPAP) treatment. OSAS appears to cause an increase in activation of CB1R in ECS. However, whether or not this increase receptor activation may result in a metabolic disorder of bone is unknown. Furthermore, it is not known whether or not rimonabant, an efficient selective $\mathrm{CB} 1 \mathrm{R}$ antagonist, can relieve the bone dysmetabolism seen in patients with OSAS.

Metabolic disorders of bone are caused by an increase in osteoclasia and a decrease in osteogenesis such that bone resorption is stronger than bone formation. Serum tartrate-resistant acid phosphatase (TRAP) is widely accepted in clinical practice and scientific research as a highly specific and sensitive index of bone metabolism reflecting changes in bone resorption [17]. Research data have indicated that the $\mathrm{CIH}$ of OSAS may affect the kidney, gastrointestinal tract, osteoblasts, osteoclasts, sympathetic nerves, leptin, and an inflammatory response resulting in abnormal bone metabolism [18, 19]. However, changes in the biochemical indexes of bone metabolism in an environment of $\mathrm{CIH}$ are not fully understood.

This research therefore aimed to establish a rat model of $\mathrm{CIH}$ in order to study the mechanism of action of the ECS in bone metabolism. This rat model then provides the opportunity to inject intraperitoneal rimonabant while measuring bone metabolism by serum TRAP levels along with examination of cell morphology in bone tissue, changes in bone microstructure, and expression of CB1R in osteocytes.

\section{Materials and method}

\section{Animal care}

Forty-eight male healthy SD rats (weighing from 450 to $500 \mathrm{~g}$ ) aged from 8 to 10 weeks were purchased from the animal center of Shanxi Medical University. Animals were maintained in clean cages with controlled temperature at $23 \pm 2{ }^{\circ} \mathrm{C}$ in $12 / 12 \mathrm{~h}$ light-dark cycles (lights on 8:00 am). They were provided free access to food and water ad libitum. All rats were weighed daily for 3 days before the experiment.

\section{CIH model}

About 48 male healthy SD rats were randomly divided into 6 groups with 8 rats in each: 2 normal control (NC) groups, 2 intermittent hypoxia (IH) groups, and $2 \mathrm{IH}+\mathrm{Ri}$ groups. Rats in NC groups were kept breathing room air for 4 weeks (4w NC group) and 6 weeks (6w NC group). Rats in IH groups experienced IH environment for 4 weeks (4w IH group) and 6 weeks ( $6 \mathrm{w}$ IH group). Apart from exposure to the same IH as in $\mathrm{IH}$ groups, rats in $\mathrm{IH}+\mathrm{Ri}$ group were given daily intraperitoneal injection of $\mathrm{Ri}$ at the dosage of $1.5 \mathrm{mg} / \mathrm{kg} / \mathrm{d}$ for 4 weeks (4w IH + Ri group) and 6 weeks $(6 \mathrm{w} \mathrm{IH}+$ $\mathrm{Ri}$ group). The IH group rats were exposed to sham or $\mathrm{CIH}$ exposure. Rats were housed as normal in standard cages placed within commercially designed environmental chambers for daily gas treatments. A gas control system was used to regulate the flow of oxygen and nitrogen into the chamber. Ambient oxygen was servocontrolled to generate intermittent hypoxia. During a 2min circle, nitrogen was first filled into the chamber at 
a set rate to reach a fraction of inspired oxygen ( $\mathrm{FiO} 2)$ at $8 \%$ from $21 \%$ within $30 \mathrm{~s}$. Then, compressed air was introduced into the chamber at a rate of $10 \mathrm{~L} / \mathrm{min}$ to achieve an $\mathrm{FiO} 2$ of $21 \%$ within $50 \mathrm{~s}$, and compressed air at $5 \mathrm{~L} / \mathrm{min}$ was filled after that to maintain the level of $21 \%$ oxygen for $40 \mathrm{~s}$ until a new cycle. Rats were placed into the chamber for 30 cycles per hour, $8 \mathrm{~h}$ per day for 4 or 6 consecutive weeks. Rats in the CG were kept in the chamber with $\mathrm{FiO} 2$ of $21 \%$ all throughout the experiment [20]. The oxygen concentration in the chambers was tested by a portable oxygen analyzer. This study was carried out in strict accordance with the recommendations in the Guide for the Care and Use of Laboratory Animals of the National Institutes of Health. The animal use protocol has been reviewed and approved by the institutional animal care and use committee of The Second Hospital of Shanxi Medical University.

Rats in the IH group received a daily intraperitoneal injection of the CB1 receptor antagonist, rimonabant $(1.5 \mathrm{mg} / \mathrm{kg} / \mathrm{d})$, a day before they were placed into the chronic intermittent low-oxygen chamber and for 4 or 6 weeks in total. The $\mathrm{NC}$ groups were exposed to only compressed air. An electrode was inserted into the cabin ventilation to confirm all changes in oxygen content. Oxygen content in the hypoxia cabin fluctuated between $8 \%$ and $21 \%$ adjusted by the gas flow-rate.

\section{Enzyme-linked immunosorbent assay (ELISA)}

After 4 or 6 weeks, the rats were anesthetized by $10 \%$ chloral hydrate, $0.3 \mathrm{ml} / 100 \mathrm{~g}$, abdominal anesthesia. Extract serum, determination of TRAP in serum by enzyme-linked immunosorbent assay. The six test tube concentrations were diluted to $160 \mathrm{pg} / \mathrm{ml}, 80 \mathrm{pg} / \mathrm{ml}$, $40 \mathrm{pg} / \mathrm{ml}, 20 \mathrm{pg} / \mathrm{ml}, 10 \mathrm{pg} / \mathrm{ml}$, and $0 \mathrm{pg} / \mathrm{ml}$. Add $50 \mathrm{ul}$ of standard samples of different concentrations to the standard wells and set blank holes and holes to be tested. Add $40 \mathrm{ul}$ of sample to the hole to be tested. Biotinylated anti-TRAP antibody was added, and blank wells were not loaded. The sampled micropores were sealed with a sealing film and placed in a $37{ }^{\circ} \mathrm{C}$ incubator for $30 \mathrm{~min}$. The washing solution was diluted 20 times and then used. Remove the sealing film, discard the liquid, add the washing solution, let stand for several tens of seconds, discard, and dry. All the wells except the blank wells were incubated with the enzyme labeling reagent and washed again. Color development, first add the color developer A50ul to each well and then add the color developer B50ul, gently shake and mix, and develop color at $37{ }^{\circ} \mathrm{C}$ for $15 \mathrm{~min}$. Termination: Blue turns yellow and the reaction is terminated, and absorbance (OD value) is measured within $15 \mathrm{~min}$.

\section{Hematoxylin and eosin staining (HE)}

After 4 or 6 weeks, the rats were anesthetized. After removing the rat tibia, fix it with $4 \%$ paraformaldehyde for $24 \mathrm{~h}$, use $4 \mathrm{~W}$ decalcifying solution, change the decalcifying solution once every 2 days until the bone tissue becomes soft, and then cut the material from the coronal plane. The alcohol gradient was dehydrated, and after the xylene was transparent, it was embedded under a paraffin machine having a melting point of $60-62{ }^{\circ} \mathrm{C}$, and finally the continuous section was about $4-5 \mu \mathrm{m}$ thick. Morphological changes in bone tissue were observed by HE staining. The dried slices were soaked in xylene, dewaxed for $10 \mathrm{~min}$ for three times, and then put into $100 \%$ and $95 \%$ alcohol, respectively, for $2 \mathrm{~min}$ with two times each. After washing with water, slices were rinsed by hematoxylin for $5 \mathrm{~min}$ and washed for three times, followed by a $1 \%$ hydrochloric acid alcohol differentiation for 1 to $2 \mathrm{~s}, 0.5 \%$ eosin coloring for $10 \mathrm{~s}$ to $1 \mathrm{~min}, 70 \%, 85 \%$ ethanol both for $2 \mathrm{~min}$, and $95 \%$ ethanol for 3 to $5 \mathrm{~s}$. After that, slices were dried over anhydrous ethanol for $1 \mathrm{~min}$ followed by gradient alcohol dehydration and the final xylene for both $2 \mathrm{~min}$ with three times each. Then slices were took out and wiped, and a drop of mounted neutral gum was added. Finally, optical microscope was used to observe the change of bone tissue morphology and structure at 100 magnifications.

\section{Immunohistochemical analysis}

The expression of $\mathrm{CB} 1$ receptor in the bone cells was detected by immunohistochemistry. The slices were dehydrated in different concentrations of ethanol for 5 min each and then put into $3 \%$ peroxide solution for $10 \mathrm{~min}$, distilled water for three times with $5 \mathrm{~min}$ each and phosphate buffer saline (PBS) liquid for 3 times with 2 min each. The 5\% albumin from bovine serum albumin (BSA) liquid is added to make the antigen closed, and the incubator is incubated for about 20 min. CB1 antibody (concentration of 1:50) is evenly added, covering the bone tissue with antibody contact, overnight at $4{ }^{\circ} \mathrm{C}$. After PBS rinsing, the second antibody placed in $37{ }^{\circ} \mathrm{C}$ incubator for $30 \mathrm{~min}$ is added. Then PBS was washed three times with 2 min each. Streptavidin-biotin complex (SABC) was joined into the wet box incubation for $20 \mathrm{~min}$, and then PBS was washed for four times. 
Diaminobenzidine (DAB) was used to color for $5 \mathrm{~min}$, to dye the extent with distilled water rinse termination. Hematoxylin staining solution was added for $15 \mathrm{~s}$, rinsed with water for $5 \mathrm{~min}$, and then put in the differentiation in hydrochloric acid alcohol. Gradient alcohol dehydration, transparent, orderly mounting was conducted under the microscope. The result showed that the negative control was only two antinegative controls. ImagePlus4.0 image analysis software was used to carry out semiquantitative analysis of and CB1 receptor in the bone tissue according to the staining area and intensity.

\section{Real-time fluorescence quantitative PCR}

Real-time fluorescence quantitative polymerase chain reaction (PCR) was also used to detect the expressions of CB1 and TRAP in bone tissues of rats. Primers were designed using Primer 3.0 software (http://frodo.wi.mit.edu/primer3). Total RNA was extracted from brain tissues of rats using Trizol reagent (Invitrogen, USA) following the manufacturers instruction. Relative mRNA expression levels were determined by SYBR Green I kit (Biotechs, China). The amplification procedure was $2 \mathrm{~min}$ at $50{ }^{\circ} \mathrm{C}$ and $10 \mathrm{~min}$ at $94{ }^{\circ} \mathrm{C}$ followed by 40 cycles of $94^{\circ} \mathrm{C}$ for $40 \mathrm{~s}, 60{ }^{\circ} \mathrm{C}$ for $30 \mathrm{~s}$, and $72{ }^{\circ} \mathrm{C}$ for $30 \mathrm{~s}$. Data were reported as values normalized by the housekeeping gene b-actin. Genetic relative quantitative was calculated with $2^{-\Delta \Delta \mathrm{Ct}}$ methods.

\section{Result}

TRAP levels in serum and bone tissues were increased by the $\mathrm{CIH}$, which was attenuated by rimonabant treatment

Although hypoxic exposure causes abnormal changes in bone metabolism, it is unclear whether $\mathrm{CIH}$ leads to bone disorder. To answer this question, we determined the levels of TRAP enzyme by ELISA in serum and bone tissues of rats exposed
Fig. 1 Determination of TRAP in serum (a) and bone tissues (b) of rats in each group by double antibody sandwich method.*IH group compared with NC group, $P<0.001$; \#6w IH group compared with 4 w IH group, $P<0.001$; @ 4w IH group compared with $4 \mathrm{w}$ IH + Ri group, $P<0.001 ; \& 6 \mathrm{w}$ IH group compared with $6 \mathrm{w} \mathrm{IH} \mathrm{+} \mathrm{Ri} \mathrm{group,}$ $P<0.001$
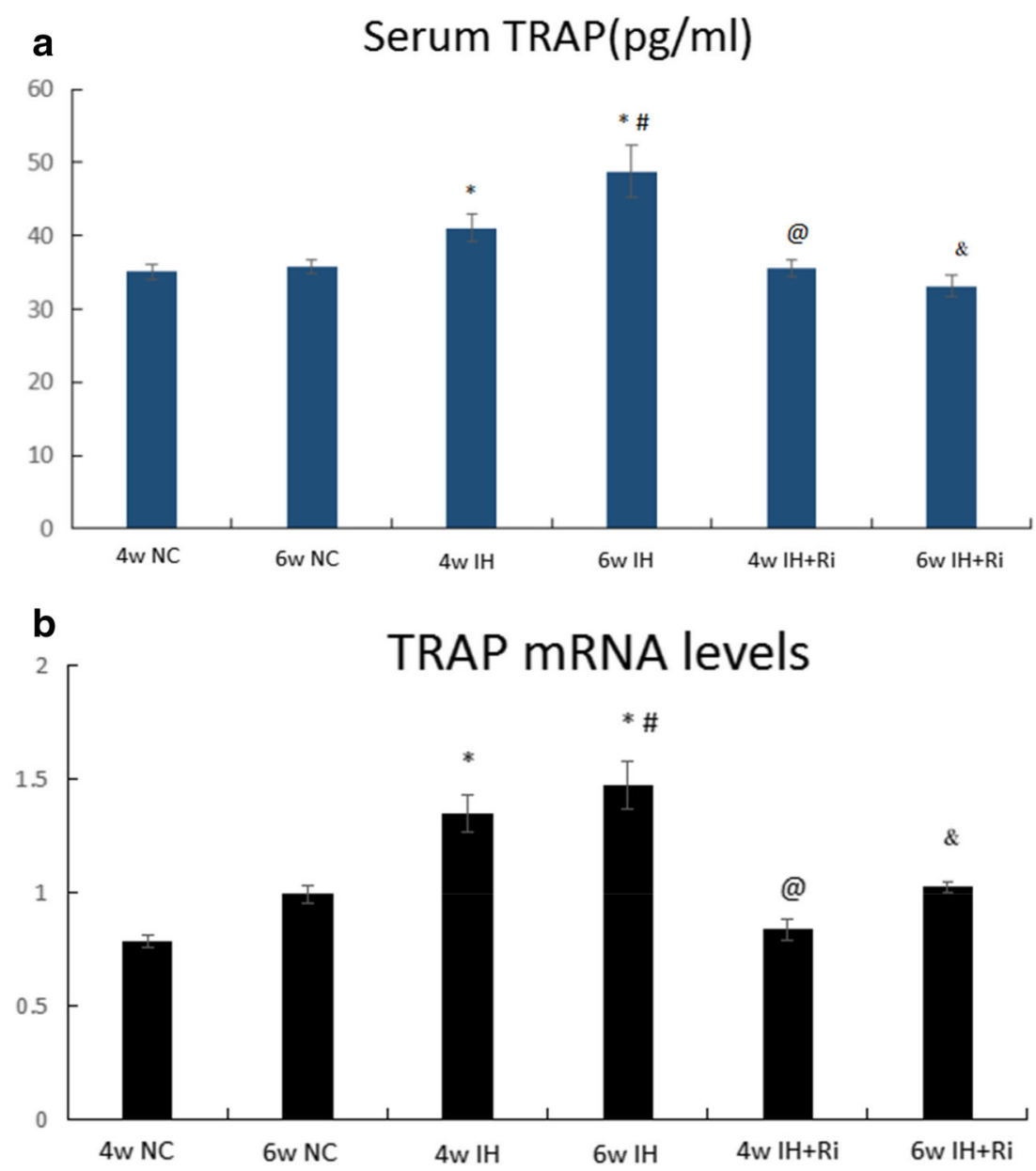
to $\mathrm{CIH}$. We found that $\mathrm{CIH}$ exposure for 4 and 6 weeks increased the levels of TRAP in both serum and bone tissues. The levels of TRAP were further increased by 6 weeks of CIH exposure compared to 4 weeks of $\mathrm{CIH}$ exposure. Treatment with rimonabant significantly reduced $\mathrm{CIH}$-induced increase in TRAP in both serum and bone tissues. Moreover, rimonabant treatment for 6 weeks was efficient to reduce the TRAP compared to 4 weeks of rimonabant treatment in response to $\mathrm{CIH}$ exposure. These results demonstrate that $\mathrm{CIH}$ exposure increases the levels of TRAP in both serum and bone tissues and these effects are reduced by CB1 antagonist rimonabant. (Fig. 1).

\section{CIH increases bone destruction in bone tissue, which is attenuated by rimonabant treatment}

We observed the morphology of rat bone by HE staining. We found that $\mathrm{CIH}$ exposure increased bone destruction at 4 and 6 weeks of exposure. The 6-week damage of $\mathrm{CIH}$ exposure was more severe than the 4-week CIH exposure. Treatment with rimonabant significantly reduced $\mathrm{CIH}$-induced bone destruction. In addition, rimonabant treatment for 6 weeks was effective in reducing bone damage compared to the 4-week rimonabant treatment group. These results indicate that $\mathrm{CIH}$ exposure increases bone tissue destruction and that the CB1 antagonist rimonabant reduces these effects (Fig. 2,bone destruction as indicated by the arrow).

\section{$\mathrm{CIH}$ increases CB1R levels in bone tissue, which is attenuated by rimonabant treatment}

We determined the levels of CB1R by immunohistochemistry in bone tissues of rats exposed to $\mathrm{CIH}$. We found that $\mathrm{CIH}$ exposure for 4 and 6 weeks increased the levels of CB1R in both serum and bone tissues. The levels of CB1R were further increased by 6 weeks of $\mathrm{CIH}$ exposure compared to 4 weeks of $\mathrm{CIH}$ exposure. Treatment with rimonabant significantly reduced $\mathrm{CIH}$-induced increase in $\mathrm{CB} 1 \mathrm{R}$ in both serum and bone tissues. Moreover, rimonabant treatment for 6 weeks was efficient to reduce the CB1R compared to 4 weeks of rimonabant treatment in response to $\mathrm{CIH}$ exposure. These results demonstrate that $\mathrm{CIH}$ exposure increases the levels of $\mathrm{CB} 1 \mathrm{R}$ in both serum and bone tissues and these effects are reduced by $\mathrm{CB} 1$ antagonist rimonabant (Figs. 3, 4, and 5).

\section{Correlation comparison}

Pearson correlation analysis showed that there was a positive correlation between ECs receptor CB1R expression level and
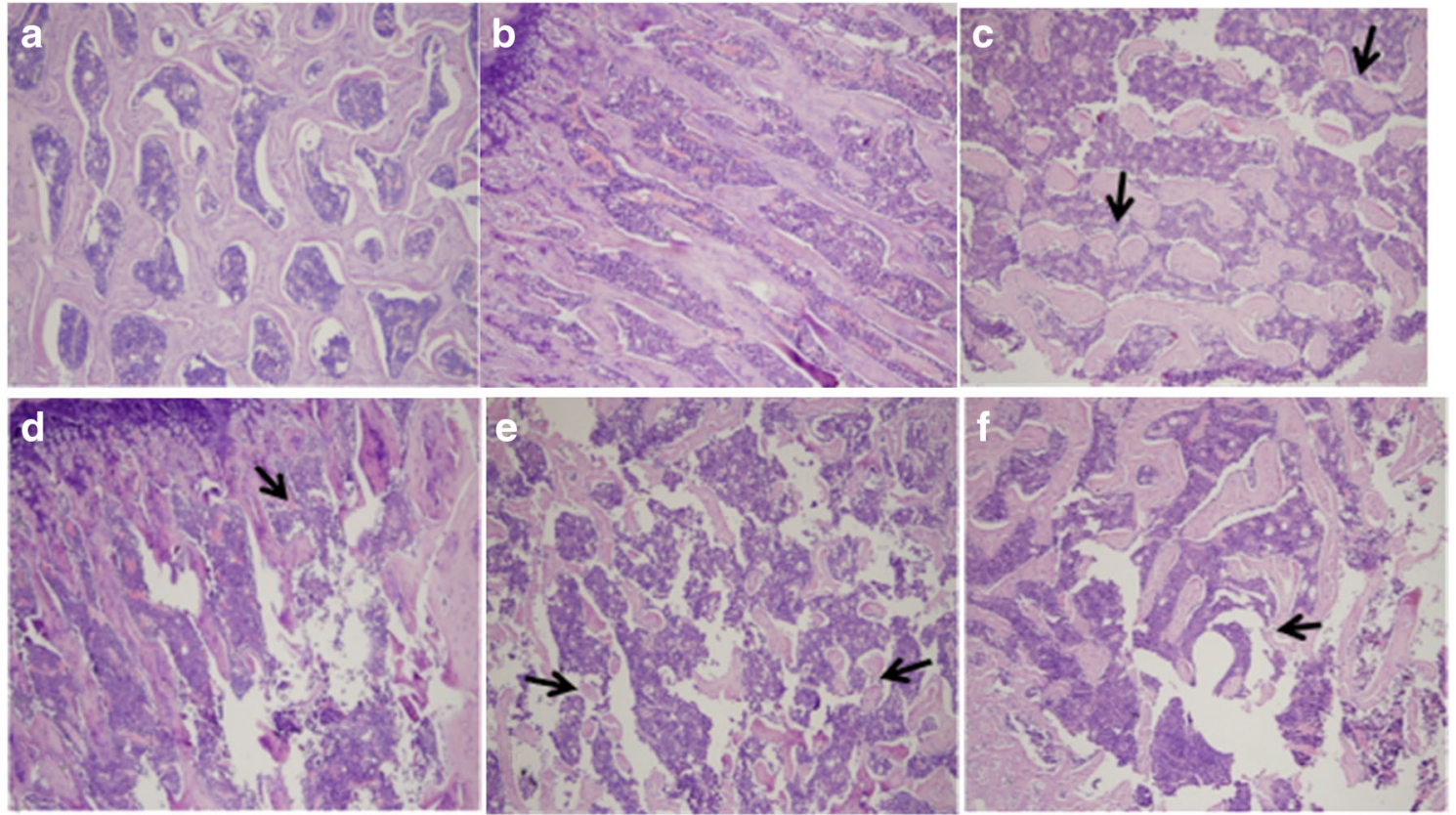

Fig. 2 Morphological changes of bone tissue in each group were observed by $\mathrm{HE}$ staining $(\mathrm{HE} \times 100)$. (a) $4 \mathrm{w} \mathrm{NC}$ group. (b) $6 \mathrm{w} \mathrm{NC}$ group. The bone tissue in $4 \mathrm{w}$ and $6 \mathrm{w}$ NC group is arranged in neat columns, and the bone trabecula structure is intact. (c) $4 \mathrm{w}$ IH group. A small amount of bone tissue is lost, their arrangement is disordered, the number is decreased, and the cells are edema. (d) 4w IH + Ri group. The trabecula structure is better than before. (e) $6 \mathrm{w} \mathrm{IH} \mathrm{group.} \mathrm{The} \mathrm{bone} \mathrm{tissue}$ loss is more serious, trabecular structure is obviously damaged, the number is significantly reduced, and bone cells are highly edema. (f) $6 \mathrm{w} \mathrm{IH}+\mathrm{Ri}$ group. The destruction of bone tissue is significantly reduced 
Fig. 3 Positive expression rate of CB1 receptor in osteoclasts (a) and osteoblasts (b) of rats in each group. *IH group compared with NC group, $P<0.001$; \#6w IH group compared with $4 \mathrm{w}$ IH group, $P<0.001$; @ 4w IH group compared with $4 \mathrm{w} \mathrm{IH}+\mathrm{Ri}$ group, $P<0.001 ; \& 6$ w IH group compared with $6 \mathrm{w} \mathrm{IH}+$ Ri group, $P<0.001$ a
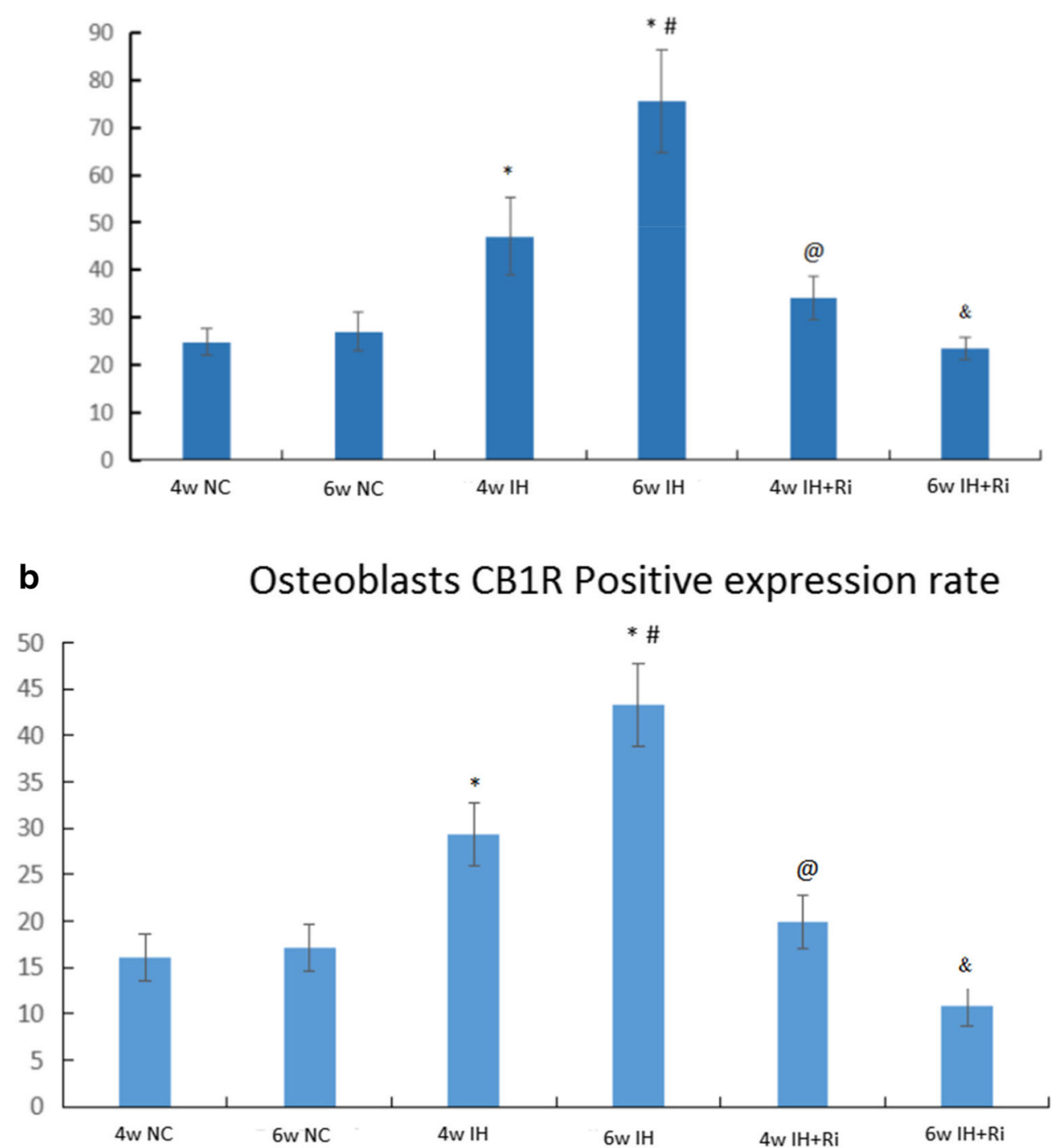

serum TRAP in OC and OB (OC/r value $0.836, P<0.001) /$ (OB/r value $0.806, P<0.001$ ) (Table 1$)$.

\section{Discussion}

In summary, the OSAS pathophysiologic process mainly involves abnormal negative pressure fluctuation in the chest, sleep structure disorder, and repeated exchange of hypoxia and reoxygenation. The most significant is $\mathrm{CIH}$ repeatedly occurring at night. All the above factors jointly play their roles and result in hyperfunction of sympathetic nerves, enhancement of systemic inflammation, and increase of oxidative stress and thus further induce the synthesis and release of a variety of inflammatory factors and active medium. Finally all the target organ systems are damaged. The epidemiological survey indicates that [21] habitual snoring disease (the common syndrome of sleep related breathing disorder) is the risk factor of fracture healing delay and OP development.

In this test, $\mathrm{CIH}$ rat model is adopted to simulate OSAS pathophysiologic process. It could basically satisfy requirements of this test. Result acquired after observing HE staining demonstrates that it can be observed using a light microscope that for rats in $\mathrm{CIH}$ environment, their bone structures develop abnormal changes, which are displayed through disordered bone trabecula structure arrangement and decrease in quantity. In addition, the degree of abnormal changes in bone structure is related to extension of time of $\mathrm{CIH}$. After using CB1R antagonist-rimonabant for intervention, abnormality of bone structures is relieved, which is displayed through that quantity 

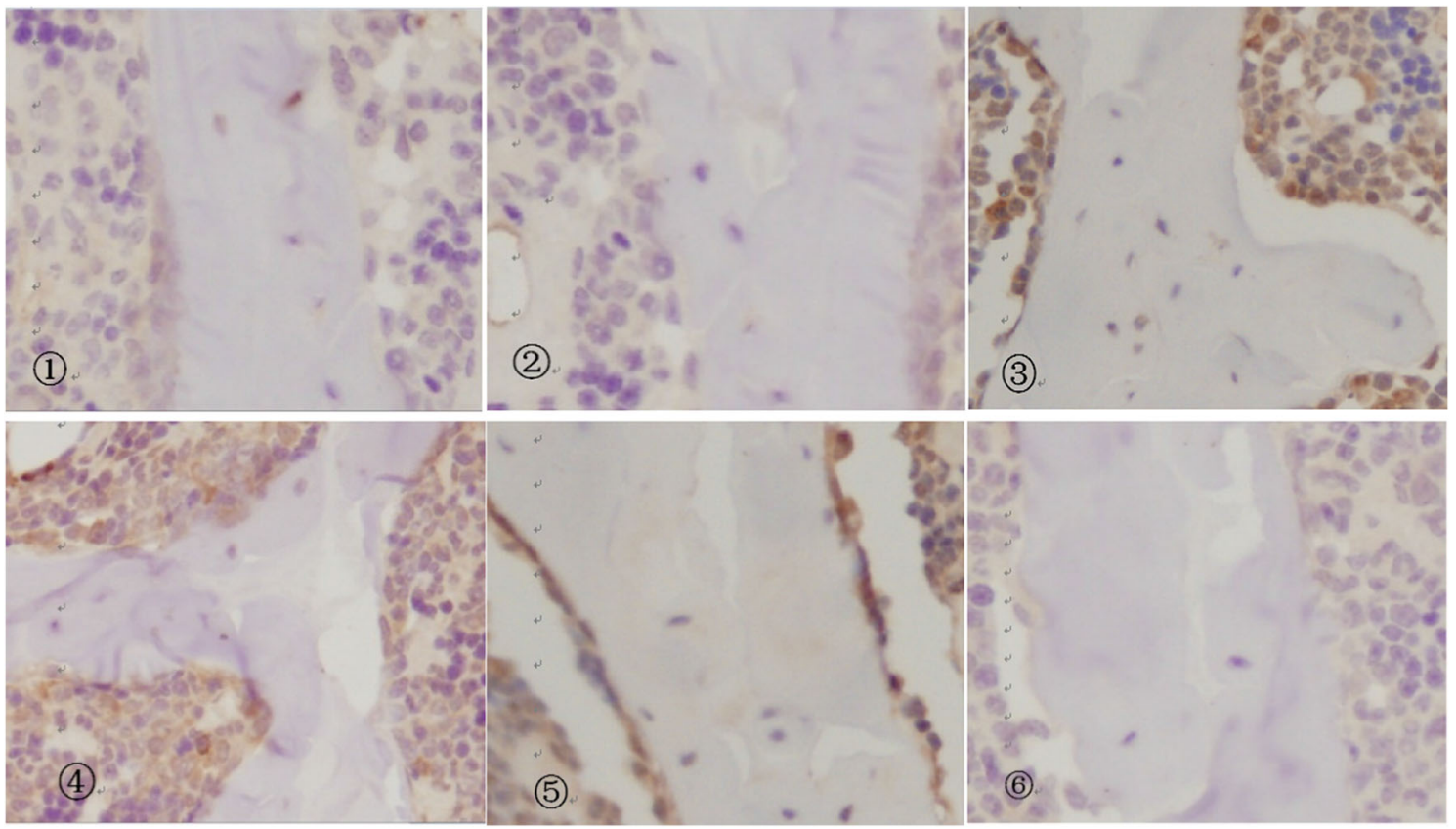

Fig. 4 Immunohistochemistry results of bone tissue in each group (immunohistochemistry, $\times 400$ ). (1), 4w NC group; (2), 6w NC group; (3), 4w IH group; (4), 4w IH + Ri group; (5), 6w IH group; (6), 6w IH + Ri group

and structure of bone trabecula have certain reserve and osteocytes only have slight swelling. Therefore, we get that such special pathological changes of OSAS in CIH may cause pathological changes in bone tissue.

That OSAS patients develop ECS disorder have been found in the early clinical study of this research group [22, 23], which is displayed through CB1R overexpression, which results in many enzymes used for synthesis of active substances and further results in increase in action of ECS. Considering that for ECS, CB1R has the receptor damaging functions, which detect the expression of CB1R to reflect changes in ECS system function. In this study, the immunohistochemical method is adopted to detect expression of $\mathrm{CB} 1 \mathrm{R}$ in $\mathrm{CIH}$ rat bone tissue. The image analysis result demonstrates that CB1R does scatter in bone tissue. Compared with normal tissue, level of expression of CB1R in IH group increases and that of $6 \mathrm{w} \mathrm{IH}$ is higher than that in $4 \mathrm{w} \mathrm{IH}$. However, in IH group where rimonabant is used for intervention, expression of CB1R decreases, and the decrease in $6 \mathrm{w}$ IH group is more obvious. There are no obvious changes among NC groups. Thus, we could know that $\mathrm{CIH}$ could increase level of expression of CB1R in bone tissue and the level of expression is related to duration of hypoxia and intervention.

Considering that specific CB1R antagonist could block combination between CB1R and its ligand, and in this test, the serum TRAP level of the bone absorption indicator has been detected. Carrying out the hypoxia intervention using
$1.5 \mathrm{mg} / \mathrm{kg} / \mathrm{d}$ rimonabant before modeling, the result indicated that after intervention, expression of CB1R was relieved and level of the serum TRAP declined. In addition, bone tissue damages were also relieved. It means rimonabant plays a role in relieving ECS disorder and bone metabolic disorder caused by $\mathrm{CIH}$ through blocking $\mathrm{CB} 1$ receptor. Meanwhile, we also carried out the correlation analysis between the level of expression of CB1R and the level of the serum TRAP, and then we found the obvious correlation. $\mathrm{CIH}$ could cause the increase in expression ratio of CB1 in osteocytes and level of the serum TRAP, as well as certain damages to bone tissue structure. Moreover, close correlation exists between them.

It is demonstrated from both sides that $\mathrm{CIH}$ caused by OSAS could cause ECS disorder and finally result in bone metabolic disorder and abnormal bone structure. Studies [24] have shown that both CB1 and CB2 receptors are expressed on mouse osteoblasts and bone marrow mesenchymal stem cells. Studies [25] have also proved that decrease in expression of CB1 receptor could relieve bone metabolic abnormality caused by ECS disorder, which is the same as the result of this test.

In short, no matter for either osteoclast or osteoblasts, $\mathrm{CIH}$ could cause the increase in expression of CB1R and further result in increase in the level of TRAP activation, which proves that $\mathrm{CIH}$ does cause ECS disorder and cause bone metabolic disorder through activating $\mathrm{CB} 1$ receptor in bone tissue. After using CB1 receptor antagonist for intervention, expression of CB1 receptor is weakened, and the level that 
Fig. 5 Positive expression rate of CB1 receptor in osteoclasts (a) and osteoblasts (b) of rats in each group.*IH group compared with NC group, $P<0.001$; \#6w IH group compared with $4 \mathrm{w}$ IH group, $P<0.001$; @ 4w IH group compared with $4 \mathrm{w} \mathrm{IH}+\mathrm{Ri}$ group, $P<0.001 ; \& 6 \mathrm{w}$ IH group compared with $6 \mathrm{w} \mathrm{IH}+$ Ri group, $P<0.001$
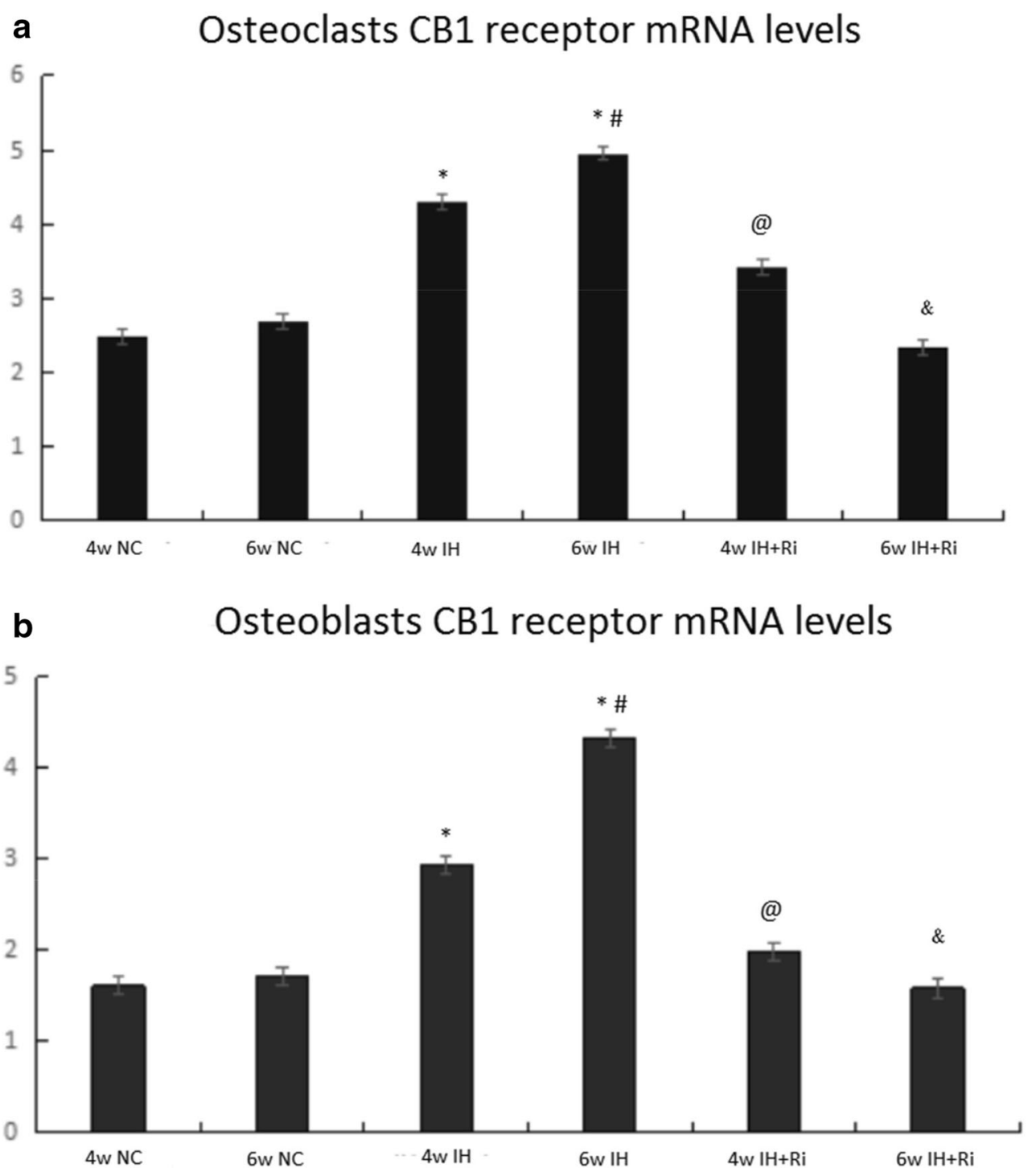

represents the bone absorption metabolic indicator also decreases. Meanwhile, damages to bone trabecula are relieved. The above indicates that the rimonabant plays a certain role in changes in bone mass and bone structure caused by $\mathrm{CIH}$ and lowers down risks of $\mathrm{CIH}$ rat model developing ECS disorder and bone metabolic abnormality and hints that effective suppression of the expression level of CB1R could provide a new way for prevention and treatment of OSAS and OP. However, we did not study changes in $\mathrm{CB} 1$ receptor and bone tissue under different hypoxia concentration, which shall be further discussed in the future study.

Table 1 Correlation between CB1R expression rate and serum TRAP levels

\begin{tabular}{lll}
\hline Index & $\mathrm{r}$ & $\mathrm{P}$ \\
\hline TRAP(OC) & 0.836 & $P<0.001$ \\
TRAP(OB) & 0.806 & $P<0.001$ \\
\hline
\end{tabular}

Note: $O B$ osteoblasts, $O C$ osteoclasts
Funding information This study was supported in part by grants from the National Natural Science Foundation of China (No. 81870076 and No. 81570086) and Research Project Supported by Shanxi Scholarship Council of China (No. 2017-120 and No. 2017-Key 6) and the International Cooperation Research of Shanxi Province Science and Technology Department (No. 201703D421027) and Health and Family Planning Commission of Shanxi Province (No. 2017048).

\section{Compliance with ethical standards}

Conflict of interest The authors declare that they have no conflict of interest.

Ethical approval The project was approved by the Ethics Committee of the Science and Technology Department of the Second Hospital of Shanxi Medical University and implemented in accordance with the Regulations of the People's Republic of China on Animal Management.

Open Access This article is licensed under a Creative Commons Attribution 4.0 International License, which permits use, sharing, adaptation, distribution and reproduction in any medium or format, as long as you give appropriate credit to the original author(s) and the source, 
provide a link to the Creative Commons licence, and indicate if changes were made. The images or other third party material in this article are included in the article's Creative Commons licence, unless indicated otherwise in a credit line to the material. If material is not included in the article's Creative Commons licence and your intended use is not permitted by statutory regulation or exceeds the permitted use, you will need to obtain permission directly from the copyright holder. To view a copy of this licence, visit http://creativecommons.org/licenses/by/4.0/.

\section{References}

1. Passali D, Corallo G, Yaremchuk S, Longini M, Proietti F, Passali GC, Bellussi L (2015) Oxidative stress in patients with obstructive sleep apnoea syndrome. Acta Otorhinolaryngol Ital 35(6):420-425. https://doi.org/10.14639/0392-100x-895

2. Toraldo DM, Den F, Deb M, Scoditti E (2015) Obstructive sleep apnoea syndrome: a new paradigm by chronic nocturnal intermittent hypoxia and sleep disruption. Acta Otorhinolaryngol Ital 35(2):69-74

3. Bibbins-Domingo K, Grossman DC, Curry SJ, Davidson KW, Epling JW Jr, Garcia FA, Herzstein J, Kemper AR, Krist AH, Kurth AE, Landefeld CS, Mangione CM, Phillips WR, Phipps MG, Pignone MP, Silverstein M, Tseng CW (2017) Screening for obstructive sleep apnea in adults: US preventive services task force recommendation statement. Jama 317(4):407-414. https://doi.org/ 10.1001/jama.2016.20325

4. Kapur VK, Auckley DH, Chowdhuri S, Kuhlmann DC, Mehra R, Ramar K, Harrod CG (2017) Clinical practice guideline for diagnostic testing for adult obstructive sleep apnea: an American Academy of sleep medicine clinical practice guideline. J Clin Sleep Med 13(3):479-504. https://doi.org/10.5664/jcsm.6506

5. Heinzer R, Vat S, Marques-Vidal P, Marti-Soler H, Andries D, Tobback N, Mooser V, Preisig M, Malhotra A, Waeber G, Vollenweider P, Tafti M, Haba-Rubio J (2015) Prevalence of sleep-disordered breathing in the general population: the HypnoLaus study. Lancet Respir Med 3(4):310-318. https://doi. org/10.1016/s2213-2600(15)00043-0

6. Subramani Y, Singh M, Wong J, Kushida CA, Malhotra A, Chung F (2017) Understanding phenotypes of obstructive sleep apnea: applications in anesthesia, surgery, and perioperative medicine. Anesth Analg 124(1):179-191. https://doi.org/10.1213/ane. 0000000000001546

7. Senaratna CV, Perret JL, Lodge CJ, Lowe AJ, Campbell BE, Matheson MC, Hamilton GS, Dharmage SC (2017) Prevalence of obstructive sleep apnea in the general population: a systematic review. Sleep Med Rev 34:70-81. https://doi.org/10.1016/j.smrv. 2016.07.002

8. Tufik S, Santos-Silva R, Taddei JA, Bittencourt LR (2010) Obstructive sleep apnea syndrome in the Sao Paulo epidemiologic sleep study. Sleep Med 11(5):441-446. https://doi.org/10.1016/j. sleep.2009.10.005

9. Reddy EV, Kadhiravan T, Mishra HK, Sreenivas V, Handa KK, Sinha S, Sharma SK (2009) Prevalence and risk factors of obstructive sleep apnea among middle-aged urban Indians: a communitybased study. Sleep Med 10(8):913-918. https://doi.org/10.1016/j. sleep.2008.08.011

10. Deis S, Srivastava RK, Ruiz de Azua I, Bindila L, Baraghithy S, Lutz B, Bab I, Tam J (2018) Age-related regulation of bone formation by the sympathetic cannabinoid CB1 receptor. Bone 108:34 42. https://doi.org/10.1016/j.bone.2017.12.018

11. Terzi R, Yilmaz Z (2016) Bone mineral density and changes in bone metabolism in patients with obstructive sleep apnea syndrome. J
Bone Miner Metab 34(4):475-481. https://doi.org/10.1007/ s00774-015-0691-1

12. McPartland JM, Guy GW, Di Marzo V (2014) Care and feeding of the endocannabinoid system: a systematic review of potential clinical interventions that upregulate the endocannabinoid system. PLoS One 9(3):e89566. https://doi.org/10.1371/journal.pone.0089566

13. Sharma C, Sadek B, Goyal SN, Sinha S, Kamal MA, Ojha S (2015) Small molecules from nature targeting G-protein coupled cannabinoid receptors: potential leads for drug discovery and development. Evid Based Complement Alternat Med 2015:238482. https://doi. org $/ 10.1155 / 2015 / 238482$

14. Sophocleous A, Marino S, Kabir D, Ralston SH, Idris AI (2017) Combined deficiency of the $\mathrm{Cnr} 1$ and $\mathrm{Cnr} 2$ receptors protects against age-related bone loss by osteoclast inhibition. Aging Cell 16(5):1051-1061. https://doi.org/10.1111/acel.12638

15. Whyte LS, Ford L, Ridge SA, Cameron GA, Rogers MJ, Ross RA (2012) Cannabinoids and bone: endocannabinoids modulate human osteoclast function in vitro. Br J Pharmacol 165(8):2584-2597. https://doi.org/10.1111/j.1476-5381.2011.01519.x

16. Smith M, Wilson R, O'Brien S, Tufarelli C, Anderson SI, O'Sullivan SE (2015) The effects of the Endocannabinoids Anandamide and 2-Arachidonoylglycerol on human osteoblast proliferation and differentiation. PLoS One 10(9):e0136546. https://doi.org/10.1371/journal.pone.0136546

17. Halling Linder C, Ek-Rylander B, Krumpel M, Norgard M, Narisawa S, Millan JL, Andersson G, Magnusson P (2017) Bone alkaline phosphatase and tartrate-resistant acid phosphatase: potential co-regulators of bone mineralization. Calcif Tissue Int 101(1): 92-101. https://doi.org/10.1007/s00223-017-0259-2

18. Bhaskara VK, Mohanam I, Gujrati M, Mohanam S (2014) Intermittent hypoxia effect on osteoclastogenesis stimulated by neuroblastoma cells. PLoS One 9(8):e105555. https://doi.org/10. 1371/journal.pone.0105555

19. Koren D, Dumin M, Gozal D (2016) Role of sleep quality in the metabolic syndrome. Diabetes Metab Syndr Obes 9:281-310. https://doi.org/10.2147/dmso.S95120

20. Gao X, Wu S, Dong Y, Huang Y, Chen Y, Qiao Y, Dou Z, Wang B (2018) Role of the endogenous cannabinoid receptor 1 in brain injury induced by chronic intermittent hypoxia in rats. Int $\mathrm{J}$ Neurosci 128(9):797-804. https://doi.org/10.1080/00207454. 2017.1420069

21. Niu J, Sahni S, Liao S, Tucker KL, Dawson-Hughes B, Gao X (2015) Association between sleep duration, insomnia symptoms and bone mineral density in older Boston Puerto Rican adults. PLoS One 10(7):e0132342. https://doi.org/10.1371/journal.pone. 0132342

22. Calik MW, Carley DW (2017) Effects of cannabinoid agonists and antagonists on sleep and breathing in Sprague-Dawley rats. Sleep 40(9). https://doi.org/10.1093/sleep/zsx112

23. Wang X, Yu Q, Yue H, Zhang J, Zeng S, Cui F (2016) Circulating Endocannabinoids and insulin resistance in patients with obstructive sleep apnea. Biomed Res Int 2016:9782031. https://doi.org/10. 1155/2016/9782031

24. Dunn SL, Wilkinson JM, Crawford A, Bunning RAD, Le Maitre CL (2016) Expression of cannabinoid receptors in human osteoarthritic cartilage: implications for future therapies. Cannabis Cannabinoid Res 1(1):3-15. https://doi.org/10.1089/can.2015.0001

25. Maccarrone M, Bab I, Biro T, Cabral GA, Dey SK, Di Marzo V, Konje JC, Kunos G, Mechoulam R, Pacher P, Sharkey KA, Zimmer A (2015) Endocannabinoid signaling at the periphery: 50 years after THC. Trends Pharmacol Sci 36(5):277-296. https://doi.org/10. 1016/j.tips.2015.02.008

Publisher's note Springer Nature remains neutral with regard to jurisdictional claims in published maps and institutional affiliations. 